NUP-A-98-7

\title{
Anomalous Gauge Theories with Antisymmetric Tensor Fields
}

\author{
Shinichi Deguchi ${ }^{a}$ 円, Tomoaki Mukai ${ }^{b}$ and Tadahito Nakajima ${ }^{b}$ \\ a) Atomic Energy Research Institute, College of Science and Technology, \\ Nihon University, Tokyo 101-8308, Japan \\ b) Department of Physics, College of Science and Technology, \\ Nihon University, Tokyo 101-8308, Japan
}

\begin{abstract}
We derive a dual theory of the four-dimensional anomalous U(1) gauge theory with a Wess-Zumino (WZ) term and with a Stückelberg type mass term by means of a duality transformation at each of the classical and quantum levels. It is shown that in the dual anomalous $\mathrm{U}(1)$ gauge theory, the $B F$ term with a rank-two antisymmetric tensor field plays the roles of the WZ term as well as the mass term of the $U(1)$ gauge field. Similar anomalous $U(1)$ gauge theory with $B F$ term is considered in six-dimensions by introducing a rank-four antisymmetric tensor field. In addition to this theory, we propose a six-dimensional anomalous $\mathrm{U}(1)$ gauge theory including an extended $B F$ term with a rank-two antisymmetric tensor field, discussing a difference between the two theories. We also consider a four-dimensional anomalous $\mathrm{SU}(2) \times \mathrm{U}(1)$ gauge theory with $B F$ term and recognize a crucial role of the $B F$ term in cancelling the non-abelian chiral anomaly.
\end{abstract}

PACS numbers: 11.15.-q, 11.30.-j, 14.70.Pw

\footnotetext{
${ }^{1}$ E-mail: deguchi@phys.cst.nihon-u.ac.jp

${ }^{2}$ E-mail: nakajima@phys.cst.nihon-u.ac.jp
} 


\section{Introduction}

The consistent quantization of anomalous gauge theories has been studied by many authors for about the past 10 years [1-6]. In particular, the chiral Schwinger model, an anomalous chiral U(1) gauge theory in two dimensions, has been investigated in detail under advantageous conditions peculiar to two dimensions. A remarkable observation is that because of the chiral anomaly, a hidden physical degree of freedom occurs in the theory [1]. This is well understood by adding a suitable Wess-Zumino (WZ) term to the classical action of the chiral Schwinger model so as to restore the gauge symmetry at the quantum level [2]. The origin of such a WZ term can be assigned to the gauge-volume integration in the path-integral quantization [3].

The idea of introducing a WZ term is, of course, applicable to anomalous gauge theories in higher dimensions. In fact, suggesting this idea, Faddeev and Shatashvili have argued for consistent quantization of an anomalous chiral gauge theory in four dimensions [4]. Since then, several authors have particularly studied the case of the $\mathrm{U}(1)$ gauge group on the reasonable assumption that the chiral gauge symmetry is spontaneously broken (via the anomaly) $[5,6]$. The symmetry breakdown makes the $\mathrm{U}(1)$ gauge field massive and enables us to adopt perturbative approaches. As an effective theory describing the broken phase, we can take the anomalous massive $\mathrm{U}(1)$ gauge theory in four dimensions whose action includes a WZ term and a Stückelberg type mass term containing a WZ scalar field [6]. This theory is consistently quantizable, though renormalizability will be spoiled owing to the WZ term.

Besides the Stückelberg formalism and the Higgs model, there is an alternative massive gauge theory called the topologically massive gauge theory, in which a topological term called the $B F$ term functions as mass terms of gauge fields $[7,8]$. The topologically massive abelian gauge theory (TMAGT) in four dimensions consists of a $\mathrm{U}(1)$ gauge field $A_{\mu}$ and a rank-two antisymmetric tensor field $B_{\mu \nu}$ [7]. It is not difficult to show, both at the classical and quantum levels, that the TMAGT is a dual version of the abelian Stückelberg formalism.

Taking this duality into account and noting that the anomalous massive U(1) gauge theory involves the abelian Stückelberg formalism, we can find a theory that is dual to the anomalous massive $\mathrm{U}(1)$ gauge theory and that involves the TMAGT. Such a dual theory will indeed be obtained in the next section with a suitable modification of the gauge transformation rule of $B_{\mu \nu}$ defined in the TMAGT. After the modification, the $B F$ term plays the roles of the WZ term as well as the mass term of $A_{\mu} ; B_{\mu \nu}$ contributes importantly to cancelling the chiral anomaly. The modified gauge transformation rule agrees with the one found in $N=1$ supergravity coupled to $N=1$ supersymmetric Maxwell theory in ten dimensions [9].

An anomaly-cancellation mechanism in which a rank-two antisymmetric tensor field plays a crucial role has been argued in superstring theories [10]. This mechanism, known as the Green-Schwarz mechanism, works only if the gauge 
group is chosen to be $\mathrm{SO}(32)$ or $\mathrm{E}_{8} \times \mathrm{E}_{8}$. A non-abelian version of the modified gauge transformation rule is also essential to the Green-Schwarz mechanism.

In this paper we would like to explain that anomaly cancellation due to an antisymmetric tensor field occurs not only in superstring theories but also in (dual versions of) ordinary anomalous gauge theories. We demonstrate that $B F$ terms and their generalizations function as WZ terms when suitable gauge transformation rules are imposed on the antisymmetric tensor fields.

The present paper is organized as follows. Section 2 derives, at the classical level, a dual theory of the anomalous massive U(1) gauge theory in four dimensions. In Section 3, the duality found in Section 2 is established at the quantum level by using the path-integral quantization based on the BRST formalism. Section 4 considers two kinds of anomalous U(1) gauge theories in six dimensions. One of them has the $B F$ term with a rank-four antisymmetric tensor field and is dual to the six-dimensional anomalous $U(1)$ gauge theory with a WZ term and with a Stückelberg type mass term. The other theory has the $B F^{2}$ term, a generalized $B F$ term, with a rank-two antisymmetric tensor field. In each theory, the six-dimensional chiral anomaly vanishes by virtue of the $B F$ or $B F^{2}$ term. Section 5 deals with an anomalous non-abelian gauge theory in four dimensions with the gauge group $\mathrm{SU}(2) \times \mathrm{U}(1)$. The $B F$ term in this theory functions both as the WZ term for the non-abelian chiral anomaly and as the mass term of the U(1) gauge field. Section 6 is devoted to summary and discussion.

\section{Dual version of an anomalous U(1) gauge the- ory}

Let us begin by discussing an anomalous massive $\mathrm{U}(1)$ gauge theory in four dimensions [6] that is defined by the lagrangian

$$
\widetilde{\mathcal{L}}=\mathcal{L}_{A}+\mathcal{L}_{\phi}+\mathcal{L}_{\mathrm{WZ}}+\mathcal{L}_{\psi}
$$

with

$$
\begin{aligned}
\mathcal{L}_{A} & =-\frac{1}{4} F_{\mu \nu} F^{\mu \nu}, \quad F_{\mu \nu} \equiv \partial_{\mu} A_{\nu}-\partial_{\nu} A_{\mu}, \\
\mathcal{L}_{\phi} & =\frac{1}{2}\left(\partial_{\mu} \phi-m A_{\mu}\right)\left(\partial^{\mu} \phi-m A^{\mu}\right) \\
\mathcal{L}_{\mathrm{WZ}} & =-\frac{k}{4} \epsilon^{\mu \nu \rho \sigma} \phi F_{\mu \nu} F_{\rho \sigma} \\
\mathcal{L}_{\psi} & =\bar{\psi} i \gamma^{\mu}\left[\partial_{\mu}-i e A_{\mu} \frac{1}{2}\left(1-\gamma_{5}\right)\right] \psi
\end{aligned}
$$

where $A_{\mu}$ is a $\mathrm{U}(1)$ gauge field, $\phi$ a WZ scalar field, $\psi$ a Dirac field, and $m$, $k$ and $e$ are constants with suitable dimensions. [Our metric has signature 
$(+,-,-,-)$. The convention for the Levi-Civita symbol is $\epsilon^{0123}=-1$. The $\gamma_{5}$ matrix is defined by $\gamma_{5}\left(=\gamma_{5}^{\dagger}\right) \equiv i \gamma^{0} \gamma^{1} \gamma^{2} \gamma^{3}$.] The second term $\mathcal{L}_{\phi}$, which is the gauge-invariant mass term in the Stückelberg formalism, is necessary for the anomalous massive $\mathrm{U}(1)$ gauge theory to make a dynamical field of $\phi$ so that perturbative analysis can be applied to the theory. The lagrangian $\widetilde{\mathcal{L}}$ then describes a massive vector field interacting with chiral fermions. The WZ term $\mathcal{L}_{\mathrm{WZ}}$ is included in $\widetilde{\mathcal{L}}$ to cancel the chiral anomaly arising from the quantum effects of $\psi$; although the lagrangian $\widetilde{\mathcal{L}}$ itself is not invariant under the gauge transformation

$$
\begin{aligned}
\delta A_{\mu} & =\partial_{\mu} \lambda \\
\delta \phi & =m \lambda \\
\delta \psi & =i e \lambda \frac{1}{2}\left(1-\gamma_{5}\right) \psi \\
\delta \bar{\psi} & =-i e \lambda \bar{\psi} \frac{1}{2}\left(1+\gamma_{5}\right)
\end{aligned}
$$

the effective action

$$
\widetilde{\Gamma}\left[A_{\mu}, \phi\right]=-i \ln \int \mathcal{D} \bar{\psi} \mathcal{D} \psi \exp \left(i \int d^{4} x \widetilde{\mathcal{L}}\right)
$$

with $k=k_{0} \equiv e^{3} /\left(24 \pi^{2} m\right)$ remains invariant due to the variation of the pathintegral measure $\mathcal{D} \bar{\psi} \mathcal{D} \psi[4]$. (Here the value of $k$ was chosen for the cancellation of the "consistent" anomaly. If we discuss the cancellation of the "covariant" anomaly, $k$ should be chosen to be $k=3 k_{0}$.) By virtue of the WZ term $\mathcal{L}_{\mathrm{WZ}}$, the $\mathrm{U}(1)$ gauge symmetry of the system holds at the quantum level. As a result, we can construct a consistent quantum field theory based on $\widetilde{\mathcal{L}}$, though this theory is power-counting nonrenormalizable owing to $\mathcal{L}_{\mathrm{WZ}}$.

To find a dual theory of the anomalous massive $\mathrm{U}(1)$ gauge theory, we now consider the first order lagrangian

$$
\mathcal{L}_{U}=-\frac{1}{6} \epsilon^{\mu \nu \rho \sigma} U_{\mu} H_{\nu \rho \sigma}+\frac{1}{2} U_{\mu} U^{\mu}+\mathcal{L}_{B F}
$$

with the so-called $B F$ term

$$
\mathcal{L}_{B F}=\frac{m}{4} \epsilon^{\mu \nu \rho \sigma} B_{\mu \nu} F_{\rho \sigma} .
$$

Here $U_{\mu}$ is a vector field, $B_{\mu \nu}$ is an antisymmetric tensor field and

$$
H_{\mu \nu \rho} \equiv F_{\mu \nu \rho}+k \omega_{\mu \nu \rho}
$$

where

$$
\begin{aligned}
F_{\mu \nu \rho} & \equiv \partial_{\mu} B_{\nu \rho}+\partial_{\nu} B_{\rho \mu}+\partial_{\rho} B_{\mu \nu}, \\
\omega_{\mu \nu \rho} & \equiv A_{\mu} F_{\nu \rho}+A_{\nu} F_{\rho \mu}+A_{\rho} F_{\mu \nu} .
\end{aligned}
$$


The tensor $\omega_{\mu \nu \rho}$ is nothing but the abelian Chern-Simons three-form. From $\mathcal{L}_{U}$ we obtain the Euler-Lagrange equation for $B_{\mu \nu}$ :

$$
\epsilon^{\mu \nu \rho \sigma} \partial_{\rho}\left(U_{\sigma}-m A_{\sigma}\right)=0
$$

which can be solved as

$$
U_{\mu}=m A_{\mu}-\partial_{\mu} \phi .
$$

Substituting (2.14) into (2.8) and removing total derivative terms, we arrive at $\mathcal{L}_{\phi}+\mathcal{L}_{\text {WZ }}$. On the other hand, the Euler-Lagrange equation for $U_{\mu}$ is

$$
U_{\mu}=\frac{1}{6} \epsilon_{\mu \nu \rho \sigma} H^{\nu \rho \sigma} .
$$

After substituting (2.15) into (2.8), we have $\mathcal{L}_{H}+\mathcal{L}_{B F}$ with

$$
\mathcal{L}_{H}=\frac{1}{12} H_{\mu \nu \rho} H^{\mu \nu \rho} \text {. }
$$

Therefore the lagrangian $\mathcal{L}_{H}+\mathcal{L}_{B F}$ is "classically" equivalent to $\mathcal{L}_{\phi}+\mathcal{L}_{\mathrm{WZ}}$.

The field strength $H_{\mu \nu \rho}$ is invariant under the gauge transformation

$$
\begin{aligned}
\delta A_{\mu} & =\partial_{\mu} \lambda, \\
\delta B_{\mu \nu} & =\partial_{\mu} \xi_{\nu}-\partial_{\nu} \xi_{\mu}-k \lambda F_{\mu \nu} .
\end{aligned}
$$

The gauge transformation rule $(2.17 \mathrm{~b})$ and the field strength $H_{\mu \nu \rho}$ agree with the ones found in $N=1$ supergravity coupled to $N=1$ supersymmetric Maxwell theory in ten dimensions [9]. Obviously $\mathcal{L}_{H}$ is gauge-invariant, whereas the topological term $\mathcal{L}_{B F}$ is not gauge-invariant and transforms as

$$
\delta \mathcal{L}_{B F}=-\frac{1}{4} m k \epsilon^{\mu \nu \rho \sigma} \lambda F_{\mu \nu} F_{\rho \sigma}+\text { total derivative. }
$$

We notice that, up to the total derivative term, the transformation behavior of $\mathcal{L}_{B F}$ is exactly the same as that of $\mathcal{L}_{\mathrm{WZ}}$. The effective action

$$
\Gamma\left[A_{\mu}, B_{\mu \nu}\right]=-i \ln \int \mathcal{D} \bar{\psi} \mathcal{D} \psi \exp \left(i \int d^{4} x \mathcal{L}\right)
$$

with the lagrangian

$$
\mathcal{L}=\mathcal{L}_{A}+\mathcal{L}_{H}+\mathcal{L}_{B F}+\mathcal{L}_{\psi}
$$

is thus gauge-invariant if $k=k_{0}$ for the consistent anomaly (or if $k=3 k_{0}$ for the covariant anomaly). The lagrangian $\mathcal{L}$ defines a dual theory of the anomalous massive $\mathrm{U}(1)$ gauge theory described by $\widetilde{\mathcal{L}}$; these two theories are equivalent (at least) at the "classical" level. (As will be seen in the next section, this equivalence persists at the "quantum" level.)

The lagrangian $\mathcal{L}_{A}+\mathcal{L}_{H}+\mathcal{L}_{B F}$ with $k=0$ is known as a starting point of the topologically massive abelian gauge theory in four dimensions [7], in which the topological term $\mathcal{L}_{B F}$ makes $A_{\mu}$ massive. Here, we would like to emphasize that in the anomalous massive $\mathrm{U}(1)$ gauge theory defined by $\mathcal{L}$, the $B F$ term $\mathcal{L}_{B F}$ plays the roles of the WZ term as well as the mass term of $A_{\mu}$. 


\section{Duality at the quantum level}

In order to complete our discussion in Section 2, we establish the equivalence of $\mathcal{L}_{H}+\mathcal{L}_{B F}$ and $\mathcal{L}_{\phi}+\mathcal{L}_{\mathrm{WZ}}$ at the "quantum" level. To this end, let us start with $\mathcal{L}_{H}+\mathcal{L}_{B F}$ and consider the covariant quantization of $B_{\mu \nu}$ using the BRST formalism [11]. We now introduce the following ghost and auxiliary fields associated with $B_{\mu \nu}$ : anticommuting vector fields $\rho_{\mu}$ and $\bar{\rho}_{\mu}$, a commuting vector field $\beta_{\mu}$, anticommuting scalar fields $\chi$ and $\bar{\chi}$, and commuting scalar fields $\sigma, \varphi$ and $\bar{\sigma}$. For our discussion, we also need an anticommuting scalar ghost field $c$ associated with $A_{\mu}$. (In what follows $A_{\mu}$ is treated as an external classical field, and so it is not necessary to introduce further ghost and auxiliary fields.) The BRST transformation $\delta$ is defined for $A_{\mu}$ and $B_{\mu \nu}$ by replacing the gauge parameters $\lambda$ and $\xi_{\mu}$ in (2.17) by the ghost fields $c$ and $\rho_{\mu}$ :

$$
\begin{aligned}
\delta A_{\mu} & =\partial_{\mu} c, \\
\delta B_{\mu \nu} & =\partial_{\mu} \rho_{\nu}-\partial_{\nu} \rho_{\mu}-k c F_{\mu \nu} .
\end{aligned}
$$

The BRST transformation rules of the other fields are defined so as to satisfy the nilpotency condition $\boldsymbol{\delta}^{2}=0$ :

$$
\begin{array}{ll}
\boldsymbol{\delta} c=0, & \\
\boldsymbol{\delta} \rho_{\mu}=-i \partial_{\mu} \sigma, & \boldsymbol{\delta} \sigma=0, \\
\boldsymbol{\delta} \bar{\rho}_{\mu}=i \beta_{\mu}, & \boldsymbol{\delta} \beta_{\mu}=0, \\
\boldsymbol{\delta} \bar{\sigma}=\bar{\chi}, & \boldsymbol{\delta} \bar{\chi}=0, \\
\boldsymbol{\delta} \varphi=\chi, & \boldsymbol{\delta} \chi=0 .
\end{array}
$$

The ghost numbers assigned to $\left(A_{\mu}, c ; B_{\mu \nu}, \rho_{\mu}, \bar{\rho}_{\mu}, \sigma, \varphi, \bar{\sigma} ; \beta_{\mu}, \chi, \bar{\chi}\right)$ and $\delta$ are $(0,1 ; 0,1,-1,2,0,-2 ; 0,1,-1)$ and 1 , respectively. To quantize $B_{\mu \nu}$, we have to introduce gauge-fixing terms with ghost number zero. Now we take the following gauge-fixing terms:

$$
\begin{gathered}
\mathcal{L}_{\mathrm{G} 1}=-i \boldsymbol{\delta}\left[B_{\mu \nu} \partial^{\mu} \bar{\rho}^{\nu}\right] \\
\mathcal{L}_{\mathrm{G} 2}=i \boldsymbol{\delta}\left[\rho^{\mu} \partial_{\mu} \bar{\sigma}+\bar{\rho}^{\mu}\left(\partial_{\mu} \varphi+u A_{\mu}+v \epsilon_{\mu \nu \rho \sigma} \omega^{\nu \rho \sigma}\right)\right]
\end{gathered}
$$

where $u$ and $v$ are gauge parameters. Because of the nilpotency of $\boldsymbol{\delta}$, these gaugefixing terms are invariant under the BRST transformation. The first term $\mathcal{L}_{\mathrm{G} 1}$ breaks the gauge invariance of $\mathcal{L}_{H}$ explicitly. The second term $\mathcal{L}_{\mathrm{G} 2}$ is necessary to break the invariance of $\mathcal{L}_{\mathrm{G} 1}$ under the secondary gauge transformation $\delta \rho_{\mu}=$ $\partial_{\mu} \varepsilon, \delta \bar{\rho}_{\mu}=\partial_{\mu} \bar{\varepsilon}$. The gauge-fixing procedure for quantization of $B_{\mu \nu}$ is complete with $\mathcal{L}_{\mathrm{G} 1}+\mathcal{L}_{\mathrm{G} 2}$. Carrying out the BRST transformation in (3.2) and (3.3), we 
obtain

$$
\begin{aligned}
\mathcal{L}_{\mathrm{G} 1}+\mathcal{L}_{\mathrm{G} 2}= & -\beta^{\nu}\left(\partial^{\mu} B_{\mu \nu}+\partial_{\nu} \varphi+u A_{\nu}+v \epsilon_{\nu \mu \rho \sigma} \omega^{\mu \rho \sigma}\right) \\
& -i \bar{\rho}^{\nu}\left(\square \rho_{\nu}-\partial_{\nu} \partial^{\mu} \rho_{\mu}-k \partial^{\mu}\left(c F_{\mu \nu}\right)\right. \\
& \left.+\partial_{\nu} \chi+u \partial_{\nu} c+3 v \epsilon_{\nu \mu \rho \sigma} F^{\mu \rho} \partial^{\sigma} c\right) \\
& -i \rho^{\nu} \partial_{\nu} \bar{\chi}-\bar{\sigma} \square \sigma+\text { total derivative }
\end{aligned}
$$

where $\square \equiv \partial_{\mu} \partial^{\mu}$.

Let us now show the quantum equivalence of $\mathcal{L}_{H}+\mathcal{L}_{B F}$ and $\mathcal{L}_{\phi}+\mathcal{L}_{\mathrm{WZ}}$. Consider the vacuum-to-vacuum amplitude

$$
Z=\int \mathcal{D} \mathcal{M} \exp \left[i \int d^{4} x\left(\mathcal{L}_{H}+\mathcal{L}_{B F}+\mathcal{L}_{\mathrm{G} 1}+\mathcal{L}_{\mathrm{G} 2}\right)\right]
$$

with the path-integral measure

$$
\mathcal{D} \mathcal{M} \equiv \mathcal{D} B_{\mu \nu} \mathcal{D} \rho_{\mu} \mathcal{D} \bar{\rho}_{\mu} \mathcal{D} \beta_{\mu} \mathcal{D} \chi \mathcal{D} \bar{\chi} \mathcal{D} \sigma \mathcal{D} \bar{\sigma} \mathcal{D} \varphi .
$$

We first notice that the integration over $\chi$ yields the delta-function $\prod_{x} \delta\left(\partial^{\nu} \bar{\rho}_{\nu}\right)$. This function enables us to remove the two terms $i \bar{\rho}^{\nu} \partial_{\nu} \partial^{\mu} \rho_{\mu}$ and $-i u \bar{\rho}^{\nu} \partial_{\nu} c$ from the exponent in (3.5). After removing them, we express the delta-function $\prod_{x} \delta\left(\partial^{\nu} \bar{\rho}_{\nu}\right)$ in the form of the integral over $\chi$ again. Then, the integration over $\rho_{\mu}$ and $\bar{\rho}_{\mu}$ yields $(\operatorname{det} \square)^{4}$. After the integrations over $\chi, \bar{\chi}, \sigma$ and $\bar{\sigma}$, the amplitude $Z$ can be written as

$$
\begin{aligned}
Z= & N_{1}(\operatorname{det} \square)^{3} \int \mathcal{D} B_{\mu \nu} \mathcal{D} \beta_{\mu} \mathcal{D} \varphi \\
& \times \exp \left[i \int d ^ { 4 } x \left\{-\frac{1}{4} B_{\mu \nu} \square B^{\mu \nu}-\frac{1}{2} B^{\nu \rho} \partial_{\rho} \partial^{\mu} B_{\mu \nu}\right.\right. \\
& -\frac{k}{2} B_{\mu \nu} \partial_{\rho} \omega^{\rho \mu \nu}+\frac{1}{12} k^{2} \omega_{\mu \nu \rho} \omega^{\mu \nu \rho}+\frac{m}{4} \epsilon^{\mu \nu \rho \sigma} B_{\mu \nu} F_{\rho \sigma} \\
& \left.\left.-\beta^{\nu}\left(\partial^{\mu} B_{\mu \nu}+\partial_{\nu} \varphi+u A_{\nu}+v \epsilon_{\nu \mu \rho \sigma} \omega^{\mu \rho \sigma}\right)\right\}\right] .
\end{aligned}
$$

Here and hereafter, $N_{i}(i=1,2,3)$ denote normalization constants. Since the integration over $\beta_{\mu}$ yields the delta-function $\prod_{x, \nu} \delta\left(\partial^{\mu} B_{\mu \nu}+\partial_{\nu} \varphi+u A_{\nu}+\right.$ $\left.v \epsilon_{\nu \mu \rho \sigma} \omega^{\mu \rho \sigma}\right)$, we replace $\partial^{\mu} B_{\mu \nu}$ in the second term of the exponent by $-\left(\partial_{\nu} \varphi+\right.$ $\left.u A_{\nu}+v \epsilon_{\nu \mu \rho \sigma} \omega^{\mu \rho \sigma}\right)$. Then, the integration over $B_{\mu \nu}$ leads to

$$
Z=N_{2} \int \mathcal{D} \beta_{\mu} \mathcal{D} \varphi \exp \left[i \int d ^ { 4 } x \left\{-\frac{1}{2} \beta_{\mu} \beta^{\mu}\right.\right.
$$




$$
\begin{aligned}
& -\beta^{\mu}\left(\partial_{\mu} \varphi+\frac{u}{2} A_{\mu}+\frac{v}{2} \epsilon_{\mu \nu \rho \sigma} \omega^{\nu \rho \sigma}\right) \\
& +\frac{1}{2}\left(\partial^{\mu} \beta_{\mu}\right) \square^{-1} \partial^{\nu}\left(-\beta_{\nu}+u A_{\nu}+v \epsilon_{\nu \lambda \rho \sigma} \omega^{\lambda \rho \sigma}\right) \\
& +\frac{1}{4}\left(\frac{1}{4} u^{2}-m^{2}\right) F_{\mu \nu} \square^{-1} F^{\mu \nu} \\
& -\epsilon^{\mu \nu \rho \sigma} F_{\mu \nu}\left(\frac{3}{8} u v F_{\rho \sigma} \square^{-1} \partial^{\lambda} A_{\lambda}\right. \\
& \left.+\frac{1}{4} m k \square^{-1} \partial^{\lambda} \omega_{\lambda \rho \sigma}\right) \\
& +\frac{1}{4} k^{2} \partial^{\rho} \omega_{\rho \mu \nu} \square^{-1} \partial_{\sigma} \omega^{\sigma \mu \nu}+\frac{1}{12}\left(k^{2}+9 v^{2}\right) \omega_{\mu \nu \rho} \omega^{\mu \nu \rho} \\
& \left.\left.-\frac{9}{32} v^{2} \epsilon^{\kappa \lambda \mu \nu} F_{\kappa \lambda} F_{\mu \nu} \square^{-1}\left(\epsilon^{\pi \rho \sigma \tau} F_{\pi \rho} F_{\sigma \tau}\right)\right\}\right] .
\end{aligned}
$$

Since the integration over $\varphi$ yields the delta-function $\prod_{x} \delta\left(\partial^{\mu} \beta_{\mu}\right)$, the terms proportional to $\partial^{\mu} \beta_{\mu}$ can be removed from the exponent in (3.8). Carrying out the integration over $\beta_{\mu}$, we obtain

$$
\begin{aligned}
Z= & N_{3} \int \mathcal{D} \varphi \exp \left[i \int d^{4} x\right. \\
& \times\left\{\frac{1}{2}\left(\partial_{\mu} \varphi+\frac{u}{2} A_{\mu}\right)\left(\partial^{\mu} \varphi+\frac{u}{2} A^{\mu}\right)\right. \\
& -\frac{3}{4} v \epsilon^{\mu \nu \rho \sigma} \varphi F_{\mu \nu} F_{\rho \sigma}+\frac{1}{4}\left(\frac{1}{4} u^{2}-m^{2}\right) F_{\mu \nu} \square^{-1} F^{\mu \nu} \\
& -\frac{1}{8}(2 m k+3 u v) \partial^{\lambda} A_{\lambda} \square^{-1}\left(\epsilon^{\mu \nu \rho \sigma} F_{\mu \nu} F_{\rho \sigma}\right) \\
& \left.\left.+\frac{1}{32}\left(k^{2}-9 v^{2}\right) \epsilon^{\kappa \lambda \mu \nu} F_{\kappa \lambda} F_{\mu \nu} \square^{-1}\left(\epsilon^{\pi \rho \sigma \tau} F_{\pi \rho} F_{\sigma \tau}\right)\right\}\right],
\end{aligned}
$$

where we have used the following formulas:

$$
\begin{aligned}
& \partial^{\rho} \omega_{\rho \mu \nu} \square^{-1} \partial_{\sigma} \omega^{\sigma \mu \nu}+\frac{1}{3} \omega_{\mu \nu \rho} \omega^{\mu \nu \rho} \\
&= \frac{1}{8} \epsilon^{\kappa \lambda \mu \nu} F_{\kappa \lambda} F_{\mu \nu} \square^{-1}\left(\epsilon^{\pi \rho \sigma \tau} F_{\pi \rho} F_{\sigma \tau}\right) \\
&+ \text { total derivative, } \\
& \epsilon^{\mu \nu \rho \sigma} \partial^{\lambda} \omega_{\lambda \mu \nu} \square^{-1} F_{\rho \sigma}= \partial^{\lambda} A_{\lambda} \square^{-1}\left(\epsilon^{\mu \nu \rho \sigma} F_{\mu \nu} F_{\rho \sigma}\right) \\
&+ \text { total derivative. }
\end{aligned}
$$

The first formula is due to the identity $\epsilon_{\kappa \lambda \mu \nu} \epsilon^{\pi \rho \sigma \tau}=-\delta_{\kappa}{ }^{[\pi} \delta_{\lambda}{ }^{\rho} \delta_{\mu}{ }^{\sigma} \delta_{\nu}{ }^{\tau]}$. The second formula is derived from $\left(\square^{-1} A_{\lambda}\right) \epsilon^{[\lambda \mu \nu \rho} \partial^{\sigma]} \partial_{\mu} \omega_{\nu \rho \sigma}=0$. (Here the brackets 
[ ] indicate a total antisymmetrization with respect to all indices put between the brackets.)

The amplitude $Z$ is independent of the gauge parameters $u$ and $v$, as might be expected; the result of functional integration in (3.9) does not include these parameters. Taking into account the gauge independence of $Z$, we now choose $u$ and $v$ to be $u=-2 m$ and $v=k / 3$ so that all the non-local terms in (3.9) can vanish. Then (3.9) becomes

$$
Z=N_{3} \int \mathcal{D} \phi \exp \left[i \int d^{4} x\left(\mathcal{L}_{\phi}+\mathcal{L}_{\mathrm{WZ}}\right)\right],
$$

where $\varphi$ has been replaced by $\phi$. Therefore the vacuum-to-vacuum amplitude based on $\mathcal{L}_{H}+\mathcal{L}_{B F}+\mathcal{L}_{\mathrm{G} 1}+\mathcal{L}_{\mathrm{G} 2}$ can be written as that based on $\mathcal{L}_{\phi}+\mathcal{L}_{\mathrm{WZ}}$, which demonstrates the equivalence of $\mathcal{L}_{H}+\mathcal{L}_{B F}$ and $\mathcal{L}_{\phi}+\mathcal{L}_{\text {WZ }}$ at the "quantum"

level. The two anomalous gauge theories characterized by $\mathcal{L}$ and $\widetilde{\mathcal{L}}$ are thus dual to each other not only at the classical level but also at the quantum level. Duality between the topologically massive abelian gauge theory and the abelian Stückelberg formalism is now obvious by setting $k=0$.

\section{Anomalous U(1) gauge theories in six dimen- sions}

We now consider the six-dimensional version of the lagrangian (2.20):

$$
\mathcal{L}_{1}^{(6)}=\mathcal{L}_{A}^{(6)}+\mathcal{L}_{H 1}^{(6)}+\mathcal{L}_{B F 1}^{(6)}+\mathcal{L}_{\psi}^{(6)},
$$

where $\mathcal{L}_{A}^{(6)}$ and $\mathcal{L}_{\psi}^{(6)}$ are the six-dimensional analogs of $\mathcal{L}_{A}$ and $\mathcal{L}_{\psi}$, respectively. The remaining two terms are given by

$$
\begin{aligned}
\mathcal{L}_{H 1}^{(6)} & =\frac{1}{2 \cdot 5 !} H_{\mu \nu \rho \sigma \tau} H^{\mu \nu \rho \sigma \tau}, \\
\mathcal{L}_{B F 1}^{(6)} & =\frac{m}{48} \epsilon^{\mu \nu \pi \rho \sigma \tau} B_{\mu \nu \pi \rho} F_{\sigma \tau}
\end{aligned}
$$

with a totally antisymmetric tensor field $B_{\mu \nu \pi \rho}$ and

$$
H_{\mu \nu \rho \sigma \tau} \equiv F_{\mu \nu \rho \sigma \tau}+\tilde{k} \omega_{\mu \nu \rho \sigma \tau},
$$

where $\tilde{k}$ is a constant and

$$
\begin{aligned}
F_{\mu \nu \rho \sigma \tau} & \equiv \frac{1}{4 !} \partial_{[\mu} B_{\nu \rho \sigma \tau]} \\
\omega_{\mu \nu \rho \sigma \tau} & \equiv \frac{1}{8} A_{[\mu} F_{\nu \rho} F_{\sigma \tau]} .
\end{aligned}
$$


[The conventions for the metric signature and the Levi-Civita symbol are $(+,-,-,-,-,-)$ and $\epsilon^{012345}=-1$.] The tensor $\omega_{\mu \nu \rho \sigma \tau}$ is known as the abelian Chern-Simons five-form. The lagrangian $\mathcal{L}_{1}^{(6)}$ describes an anomalous massive $\mathrm{U}(1)$ gauge theory in six dimensions. Since the field strength $H_{\mu \nu \rho \sigma \tau}$ is invariant under the gauge transformation

$$
\begin{aligned}
\delta A_{\mu} & =\partial_{\mu} \lambda \\
\delta B_{\mu \nu \rho \sigma} & =\frac{1}{6} \partial_{[\mu} \xi_{\nu \rho \sigma]}-\frac{1}{8} \tilde{k} \lambda F_{[\mu \nu} F_{\rho \sigma]},
\end{aligned}
$$

$\mathcal{L}_{H 1}^{(6)}$ is also invariant, while the six-dimensional $B F$ term $\mathcal{L}_{B F 1}^{(6)}$ transforms as

$$
\begin{aligned}
\delta \mathcal{L}_{B F 1}^{(6)}= & -\frac{1}{16} m \tilde{k} \epsilon^{\mu \nu \pi \rho \sigma \tau} \lambda F_{\mu \nu} F_{\pi \rho} F_{\sigma \tau} \\
& + \text { total derivative. }
\end{aligned}
$$

This transformation behavior is essential to cancellation of the six-dimensional chiral anomaly due to the quantum effects of the Dirac field $\psi$; the six-dimensional analog of (2.19) with the lagrangian $\mathcal{L}_{1}^{(6)}$ is gauge-invariant, if $\tilde{k}$ is chosen to be $\tilde{k}=\tilde{k}_{0} \equiv e^{4} /\left(96 \pi^{3} m\right)$ for the consistent anomaly (or $\tilde{k}=4 \tilde{k}_{0}$ for the covariant anomaly).

Similarly to the case of four dimensions, we can represent $\mathcal{L}_{H 1}^{(6)}+\mathcal{L}_{B F 1}^{(6)}$ as $\mathcal{L}_{\phi}^{(6)}+\mathcal{L}_{\mathrm{WZ}}^{(6)}$ by means of a duality transformation at each of the classical and quantum levels, where $\mathcal{L}_{\phi}^{(6)}$ is the six-dimensional analog of $(2.3)$ and $\mathcal{L}_{\mathrm{WZ}}^{(6)}$ is the WZ term in six dimensions:

$$
\mathcal{L}_{\mathrm{WZ}}^{(6)}=-\frac{\tilde{k}}{16} \epsilon^{\mu \nu \pi \rho \sigma \tau} \phi F_{\mu \nu} F_{\pi \rho} F_{\sigma \tau} .
$$

The gauge theory defined by $\mathcal{L}_{1}^{(6)}$ is dual to the anomalous massive $\mathrm{U}(1)$ gauge theory defined by the lagrangian $\mathcal{L}_{A}^{(6)}+\mathcal{L}_{\phi}^{(6)}+\mathcal{L}_{\mathrm{WZ}}^{(6)}+\mathcal{L}_{\psi}^{(6)}$.

In six dimensions, we can also consider an anomalous $\mathrm{U}(1)$ gauge theory with a topological term consisting of $F_{\mu \nu}$ and, instead of $B_{\mu \nu \rho \sigma}$, an antisymmetric tensor field $B_{\mu \nu}$ obeying the gauge transformation rule (2.17b). This theory is characterized by the lagrangian

$$
\mathcal{L}_{2}^{(6)}=\mathcal{L}_{A}^{(6)}+\mathcal{L}_{H 2}^{(6)}+\mathcal{L}_{B F 2}^{(6)}+\mathcal{L}_{\psi}^{(6)}
$$

Here $\mathcal{L}_{H 2}^{(6)}$ has the same form as $(2.16)$, and $\mathcal{L}_{B F 2}^{(6)}$ is the $B F^{2}$ term, a generalized $B F$ term,

$$
\mathcal{L}_{B F 2}^{(6)}=\frac{1}{16} l^{2} \epsilon^{\mu \nu \pi \rho \sigma \tau} B_{\mu \nu} F_{\pi \rho} F_{\sigma \tau},
$$


where $l$ is a constant. Under the gauge transformation $(2.17), \mathcal{L}_{B F 2}^{(6)}$ transforms in a similar manner to $\mathcal{L}_{B F 1}^{(6)}$, namely

$$
\begin{aligned}
\delta \mathcal{L}_{B F 2}^{(6)}= & -\frac{1}{16} l^{2} k \epsilon^{\mu \nu \pi \rho \sigma \tau} \lambda F_{\mu \nu} F_{\pi \rho} F_{\sigma \tau} \\
& + \text { total derivative }
\end{aligned}
$$

Hence, the six-dimensional analog of (2.19) with the lagrangian $\mathcal{L}_{2}^{(6)}$ is also gauge-invariant, if $k$ is chosen to be $k=\bar{k}_{0} \equiv e^{4} /\left(96 \pi^{3} l^{2}\right)$ for the consistent anomaly (or $k=4 \bar{k}_{0}$ for the covariant anomaly).

The two anomalous gauge theories defined by $\mathcal{L}_{1}^{(6)}$ and $\mathcal{L}_{2}^{(6)}$ are substantially different. The lagrangian $\mathcal{L}_{1}^{(6)}$ describes a massive vector field interacting with chiral fermions; the only physical degree of freedom of $B_{\mu \nu \rho \sigma}$ is observed as the longitudinal mode of the massive vector field. The $B F$ term $\mathcal{L}_{B F 1}^{(6)}$ functions both as the WZ term and as the mass term of $A_{\mu}$. In contrast, the lagrangian $\mathcal{L}_{2}^{(6)}$ describes a massless vector field interacting with chiral fermions and with a massless rank-two antisymmetric tensor field. All the physical degrees of freedom of $B_{\mu \nu}$ are observed as its own massless modes. Although $\mathcal{L}_{B F 2}^{(6)}$ plays the role of the WZ term, it does not function as a mass term.

It has been shown that in the two theories defined by $\mathcal{L}_{1}^{(6)}$ and $\mathcal{L}_{2}^{(6)}$, the sixdimensional chiral anomaly vanishes by virtue of the topological terms $\mathcal{L}_{B F 1}^{(6)}$ and $\mathcal{L}_{B F 2}^{(6)}$ with suitable coefficients. We can thus construct consistent quantum field theories based on $\mathcal{L}_{1}^{(6)}$ and $\mathcal{L}_{2}^{(6)}$.

In higher dimensions, the varieties of generalized $B F$ terms increase: for example, in $2 n$ dimensions, we can construct a $B F$ term and $n-2$ generalized $B F$ terms,

$$
\begin{aligned}
& \epsilon^{\mu_{1} \cdots \mu_{2 p} \mu_{2 p+1} \mu_{2 p+2} \cdots \mu_{2 n-1} \mu_{2 n}} \\
& \times B_{\mu_{1} \cdots \mu_{2 p}} F_{\mu_{2 p+1} \mu_{2 p+2}} \cdots F_{\mu_{2 n-1} \mu_{2 n}}, \\
& \quad(p=1,2,3, \ldots, n-1)
\end{aligned}
$$

where $B_{\mu_{1} \cdots \mu_{2 p}}$ is a totally antisymmetric tensor field of rank $2 p$. Imposing an appropriate gauge transformation rule such as (2.17b) and (4.6b) on $B_{\mu_{1} \cdots \mu_{2 p}}$, we can make the $B F$ and generalized $B F$ terms (4.12) function as the WZ term for the chiral $\mathrm{U}(1)$ anomaly in $2 n$ dimensions.

\section{Anomalous non-abelian gauge theory}

We next discuss an anomalous non-abelian gauge theory in four dimensions whose gauge group is $\mathrm{SU}(2) \times \mathrm{U}(1)$. Introducing a $\mathrm{U}(1)$ gauge field $A_{\mu}$, a $\mathrm{SU}(2)$ gauge field $\widehat{A}_{\mu}=\frac{1}{2} \widehat{A}_{\mu}{ }^{a} \sigma_{a}\left[\sigma_{a}(a=1,2,3)\right.$ denote the Pauli matrices $]$, and a 
Dirac field $\widehat{\psi}$ belonging to the fundamental representation of $\mathrm{SU}(2)$, we consider the lagrangian $\widehat{\mathcal{L}}_{A}+\widehat{\mathcal{L}}_{\psi}$ with

$$
\begin{aligned}
& \widehat{\mathcal{L}}_{A}=-\frac{1}{4} F_{\mu \nu} F^{\mu \nu}-\frac{1}{4} \operatorname{tr}\left[\widehat{F}_{\mu \nu} \widehat{F}^{\mu \nu}\right], \\
& \widehat{\mathcal{L}}_{\psi}=\overline{\bar{\psi}} i \gamma^{\mu}\left[\partial_{\mu}-i\left(e A_{\mu} \sigma_{0}+g \widehat{A}_{\mu}\right) \frac{1}{2}\left(1-\gamma_{5}\right)\right] \widehat{\psi},
\end{aligned}
$$

where

$$
\begin{aligned}
& F_{\mu \nu} \equiv \partial_{\mu} A_{\nu}-\partial_{\nu} A_{\mu} \\
& \widehat{F}_{\mu \nu} \equiv \partial_{\mu} \widehat{A}_{\nu}-\partial_{\nu} \widehat{A}_{\mu}-i g\left[\widehat{A}_{\mu}, \widehat{A}_{\nu}\right],
\end{aligned}
$$

$\sigma_{0}$ is the $2 \times 2$ unit matrix, and $e$ and $g$ are constants. The lagrangian $\widehat{\mathcal{L}}_{A}+\widehat{\mathcal{L}}_{\psi}$ is invariant under the gauge transformation

$$
\begin{aligned}
\delta A_{\mu} & =\partial_{\mu} \lambda \\
\delta \widehat{A}_{\mu} & =\partial_{\mu} \widehat{\lambda}-i g\left[\widehat{A}_{\mu}, \widehat{\lambda}\right], \\
\delta \widehat{\psi} & =i\left(e \lambda \sigma_{0}+g \widehat{\lambda}\right) \frac{1}{2}\left(1-\gamma_{5}\right) \widehat{\psi}, \\
\delta \overline{\widehat{\psi}} & =-i \overline{\widehat{\psi}}\left(e \lambda \sigma_{0}+g \widehat{\lambda}\right) \frac{1}{2}\left(1+\gamma_{5}\right),
\end{aligned}
$$

where $\widehat{\lambda}$ is represented as $\widehat{\lambda}=\frac{1}{2} \widehat{\lambda}^{a} \sigma_{a}$.

In the chiral gauge theory defined by $\widehat{\mathcal{L}}_{A}+\widehat{\mathcal{L}}_{\psi}$, a non-abelian chiral anomaly arises necessarily due to the quantum effects of $\widehat{\psi}$. We can find the anomaly in the gauge transformation of the effective action $W\left[A_{\mu}, \widehat{A}_{\mu}\right]$ defined from the path-integral of $\exp \left(i \int d^{4} x \widehat{\mathcal{L}}_{\psi}\right)$ over $\overline{\widehat{\psi}}$ and $\widehat{\psi}$. The gauge transformation of $W$ is systematically calculated using the perturbative or non-perturbative method with suitable regularization procedures for ill-defined quantities [12]. Adopting a certain regularization procedure, we obtain the "consistent" anomaly; in the case at hand, it can be written as

$$
\begin{aligned}
\delta W= & \int d^{4} x \frac{1}{24 \pi^{2}} \epsilon^{\mu \nu \rho \sigma} \\
& \times \operatorname{tr}\left[\Lambda \partial_{\mu}\left(\mathcal{A}_{\nu} \partial_{\rho} \mathcal{A}_{\sigma}-\frac{i}{2} \mathcal{A}_{\nu} \mathcal{A}_{\rho} \mathcal{A}_{\sigma}\right)\right] \\
= & \int d^{4} x \frac{1}{24 \pi^{2}} \epsilon^{\mu \nu \rho \sigma}\left\{\frac{1}{2} e^{3} \lambda F_{\mu \nu} F_{\rho \sigma}\right. \\
& +e g^{2} F_{\mu \nu} \operatorname{tr}\left[\widehat{\lambda} \partial_{\rho} \widehat{A}_{\sigma}\right]-\frac{i}{2} e g^{3} \operatorname{tr}\left[\widehat{\lambda}_{\mu}\left(A_{\nu} \widehat{A}_{\rho} \widehat{A}_{\sigma}\right)\right] \\
& +e g^{2} \lambda \operatorname{tr}\left[\partial_{\mu}\left(\widehat{A}_{\nu} \partial_{\rho} \widehat{A}_{\sigma}-\frac{i}{2} g \widehat{A}_{\nu} \widehat{A}_{\rho} \widehat{A}_{\sigma}\right)\right] \\
& \left.+g^{3} \operatorname{tr}\left[\widehat{\lambda} \partial_{\mu}\left(\widehat{A}_{\nu} \partial_{\rho} \widehat{A}_{\sigma}-\frac{i}{2} g \widehat{A}_{\nu} \widehat{A}_{\rho} \widehat{A}_{\sigma}\right)\right]\right\},
\end{aligned}
$$


where $\mathcal{A}_{\mu} \equiv e A_{\mu} \sigma_{0}+g \widehat{A}_{\mu}$ and $\Lambda \equiv e \lambda \sigma_{0}+g \widehat{\lambda}$. The term in the last line of (5.6) vanishes because of a property of the Pauli matrices. The remainder of the integrand can be expressed as

$$
\begin{aligned}
& \frac{1}{24 \pi^{2}} \epsilon^{\mu \nu \rho \sigma}\left\{\frac{1}{2} e^{3} \lambda F_{\mu \nu} F_{\rho \sigma}+\frac{3}{2} e g^{2} F_{\mu \nu} \operatorname{tr}\left[\widehat{\lambda} \partial_{\rho} \widehat{A}_{\sigma}\right]\right\} \\
& -\delta w+\text { total derivative }
\end{aligned}
$$

with

$$
w \equiv \frac{1}{24 \pi^{2}} e g^{2} \epsilon^{\mu \nu \rho \sigma} A_{\mu} \operatorname{tr}\left[\widehat{A}_{\nu} \partial_{\rho} \widehat{A}_{\sigma}-\frac{i}{2} g \widehat{A}_{\nu} \widehat{A}_{\rho} \widehat{A}_{\sigma}\right] .
$$

In (5.7), $-\delta w$ is considered to be a "trivial" violation of gauge symmetry, since it can be removed by adding the "local" functional $\int d^{4} x w$ to the effective action $W$. (The trivial violation $-\delta w$ is due to the regularization procedure adopted here, and so does not alter the anomalous content of the theory.) We thus arrive at the anomaly written in the following form:

$$
\begin{aligned}
\delta\left(W+\int d^{4} x w\right) \\
=\int d^{4} x \frac{1}{24 \pi^{2}} \epsilon^{\mu \nu \rho \sigma}\left\{\frac{1}{2} e^{3} \lambda F_{\mu \nu} F_{\rho \sigma}\right. \\
\left.\quad+\frac{3}{2} e g^{2} F_{\mu \nu} \operatorname{tr}\left[\widehat{\lambda} \partial_{\rho} \widehat{A}_{\sigma}\right]\right\} .
\end{aligned}
$$

The right-hand side of (5.9) can never be written in the form of the gauge variation of a local functional only in $A_{\mu}$ and $\widehat{A}_{\mu}$. For this reason, we can not find a gauge-invariant effective action without introducing extra physical degrees of freedom.

In order that the gauge symmetry may be restored to the theory, let us introduce the $B F$ term (2.9) with $B_{\mu \nu}$ obeying the gauge transformation rule

$$
\delta B_{\mu \nu}=\partial_{\mu} \xi_{\nu}-\partial_{\nu} \xi_{\mu}-k \lambda F_{\mu \nu}-\widehat{k} \operatorname{tr}\left[\widehat{\lambda} \partial_{[\mu} \widehat{A}_{\nu]}\right]
$$

instead of (2.17b). Here $k$ and $\widehat{k}$ are constants. At the same time, we modify the field strength $H_{\mu \nu \rho}$ in (2.10) so it is invariant under the gauge transformation defined by (5.5) and (5.10). The field strength modified satisfactorily is found to be

$$
\widehat{H}_{\mu \nu \rho} \equiv F_{\mu \nu \rho}+k \omega_{\mu \nu \rho}+\widehat{k} \widehat{\omega}_{\mu \nu \rho}
$$

where $F_{\mu \nu \rho}$ and $\omega_{\mu \nu \rho}$ have been given in (2.11) and (2.12), and $\widehat{\omega}_{\mu \nu \rho}$ is the non-abelian Chern-Simons three-form

$$
\widehat{\omega}_{\mu \nu \rho} \equiv \operatorname{tr}\left[\widehat{A}_{[\mu} \partial_{\nu} \widehat{A}_{\rho]}-\frac{2}{3} i g \widehat{A}_{[\mu} \widehat{A}_{\nu} \widehat{A}_{\rho]}\right] \text {. }
$$


The gauge transformation rule (5.10) and the field strength $\widehat{H}_{\mu \nu \rho}$ were first found by Chapline and Manton in the study of $N=1$ supergravity coupled to $N=1$ supersymmetric Yang-Mills theory in ten dimensions [13]. Recently, (5.10) and $\widehat{H}_{\mu \nu \rho}$ have also been obtained in the Yang-Mills theory in loop space with the affine gauge group [14]. Since $\widehat{H}_{\mu \nu \rho}$ is gauge-invariant,

$$
\widehat{\mathcal{L}}_{H}=\frac{1}{12} \widehat{H}_{\mu \nu \rho} \widehat{H}^{\mu \nu \rho}
$$

is also invariant, while the $B F$ term (2.9) transforms as

$$
\begin{aligned}
\delta \mathcal{L}_{B F}= & -\frac{1}{4} m \epsilon^{\mu \nu \rho \sigma}\left(k \lambda F_{\mu \nu} F_{\rho \sigma}+2 \widehat{k} F_{\mu \nu} \operatorname{tr}\left[\widehat{\lambda} \partial_{\rho} \widehat{A}_{\sigma}\right]\right) \\
& + \text { total derivative. }
\end{aligned}
$$

Comparing (5.14) with (5.9), we see that the non-abelian analog of (2.19) with the lagrangian

$$
\widehat{\mathcal{L}}=\widehat{\mathcal{L}}_{A}+\widehat{\mathcal{L}}_{H}+\mathcal{L}_{B F}+\widehat{\mathcal{L}}_{\psi}
$$

is gauge-invariant (up to the trivial violation $-\delta w$ ), if $k$ and $\widehat{k}$ are chosen to be $k=e^{3} /\left(12 \pi^{2} m\right)$ and $\widehat{k}=e g^{2} /\left(8 \pi^{2} m\right)$. In this case, the $B F$ term $\mathcal{L}_{B F}$ plays the roles of the WZ term for the non-abelian chiral anomaly as well as the mass term of $A_{\mu}$. Consequently, the $\mathrm{SU}(2) \times \mathrm{U}(1)$ gauge symmetry is restored at the quantum level, though the $\mathrm{U}(1)$ gauge field $A_{\mu}$ becomes massive. (The $\mathrm{SU}(2)$ gauge field $\widehat{A}_{\mu}$ remains massless.) We can thus construct a consistent quantum theory based on $\widehat{\mathcal{L}}$.

Through the first order lagrangian that is defined by replacing $H_{\mu \nu \rho}$ in (2.8) with $\widehat{H}_{\mu \nu \rho}$, we can easily verify the classical equivalence of $\widehat{\mathcal{L}}_{H}+\mathcal{L}_{B F}$ and

$$
\mathcal{L}^{\prime}=\mathcal{L}_{\phi}+\widehat{\mathcal{L}}_{\mathrm{WZ}}-\frac{1}{6} m \widehat{k} \epsilon^{\mu \nu \rho \sigma} A_{\mu} \widehat{\omega}_{\nu \rho \sigma}
$$

where $\mathcal{L}_{\phi}$ is given by $(2.3)$ and $\widehat{\mathcal{L}_{\mathrm{WZ}}}$ is the WZ term

$$
\widehat{\mathcal{L}}_{\mathrm{WZ}}=-\frac{1}{4} \epsilon^{\mu \nu \rho \sigma} \phi\left(k F_{\mu \nu} F_{\rho \sigma}+\widehat{k} \operatorname{tr}\left[\widehat{F}_{\mu \nu} \widehat{F}_{\rho \sigma}\right]\right) .
$$

The equivalence of $\widehat{\mathcal{L}}_{H}+\mathcal{L}_{B F}$ and $\mathcal{L}^{\prime}$ holds also at the quantum level. To show this, it is necessary to introduce an anticommuting scalar ghost field $\widehat{c}=\frac{1}{2} \widehat{c}^{a} \sigma_{a}$ associated with $\widehat{A}_{\mu}$ in addition to the ghost and auxiliary fields introduced in Section 3. From the gauge transformation rules (5.5a), (5.5b) and (5.10), the BRST transformation rules of $A_{\mu}, \widehat{A}_{\mu}$ and $B_{\mu \nu}$ are determined to be

$$
\begin{aligned}
\boldsymbol{\delta} A_{\mu} & =\partial_{\mu} c \\
\boldsymbol{\delta} \widehat{A}_{\mu} & =\partial_{\mu} \widehat{c}-i g\left[\widehat{A}_{\mu}, \widehat{c}\right] \\
\boldsymbol{\delta} B_{\mu \nu} & =\partial_{\mu} \rho_{\nu}-\partial_{\nu} \rho_{\mu}-k c F_{\mu \nu}-\widehat{k} \operatorname{tr}\left[\widehat{c} \partial_{[\mu} \widehat{A}_{\nu]}\right] .
\end{aligned}
$$


Here the ghost number 1 has been assigned to $\widehat{c}$. The BRST transformation rule of $\widehat{c}$ is determined from the nilpotency of $\boldsymbol{\delta}$, which also demands some modifications of the BRST transformation rules of $\rho_{\mu}$ and $\sigma$ given in (3.1b) [15]. We thus have

$$
\begin{aligned}
\boldsymbol{\delta} \widehat{c} & =i g \widehat{c}^{2}, \\
\boldsymbol{\delta} \rho_{\mu} & =-i \partial_{\mu} \sigma-i g \widehat{k} \operatorname{tr}\left[\widehat{c}^{2} \widehat{A}_{\mu}\right] \\
\boldsymbol{\delta} \sigma & =-\frac{1}{3} g \widehat{k} \operatorname{tr}\left[\widehat{c}^{3}\right] .
\end{aligned}
$$

The other ghost and auxiliary fields obey the transformation rules in (3.1b). Now we take the gauge-fixing terms (3.2) and, instead of (3.3),

$$
\begin{aligned}
\widehat{\mathcal{L}}_{\mathrm{G} 2}= & i \boldsymbol{\delta}\left[\rho^{\mu} \partial_{\mu} \bar{\sigma}+\bar{\rho}^{\mu}\left(\partial_{\mu} \varphi+u A_{\mu}\right.\right. \\
& \left.\left.+v \epsilon_{\mu \nu \rho \sigma} \omega^{\nu \rho \sigma}+\widehat{v} \epsilon_{\mu \nu \rho \sigma} \widehat{\omega}^{\nu \rho \sigma}\right)\right]
\end{aligned}
$$

with the gauge parameters $u, v$ and $\widehat{v}$. Starting with the vacuum-to-vacuum amplitude, $\widehat{Z}$, with the lagrangian $\widehat{\mathcal{L}}_{H}+\mathcal{L}_{B F}+\mathcal{L}_{\mathrm{G} 1}+\widehat{\mathcal{L}}_{\mathrm{G} 2}$ and by following the same procedure that we used in Section 3 , we can rewrite $\widehat{Z}$ as the vacuum-tovacuum amplitude with a lagrangian consisting of certain local and non-local terms. Since the amplitude $\widehat{Z}$ is independent of the gauge parameters, we choose them to be $u=-2 m, v=k / 3$ and $\widehat{v}=\widehat{k} / 3$; then all the non-local terms vanish and $\mathcal{L}^{\prime}$ alone remains as a lagrangian defining $\widehat{Z}$. This result demonstrates the quantum equivalence of $\widehat{\mathcal{L}}_{H}+\mathcal{L}_{B F}$ and $\mathcal{L}^{\prime}$. Needless to say, the lagrangian $\widehat{\mathcal{L}}_{A}+\mathcal{L}^{\prime}+\widehat{\mathcal{L}}_{\psi}$ defines a dual theory of the anomalous gauge theory described by $\widehat{\mathcal{L}}$.

Adding a gauge variation of any local functional in gauge fields to an anomaly does not alter the anomalous content of the theory. In other words, an anomaly is unique up to gauge variations of local functionals in gauge fields, and so may take various forms. In the theory under consideration, adding the local functional $\int d^{4} x\left(w-\frac{1}{6} m \widehat{k} \epsilon^{\mu \nu \rho \sigma} A_{\mu} \widehat{\omega}_{\nu \rho \sigma}\right)$ to the effective action $W$, we obtain a form of the anomaly that is completely canceled with the gauge variation of the WZ action $\int d^{4} x \widehat{\mathcal{L}}_{\mathrm{WZ}}$. Hence, it is possible to construct a consistent quantum theory based on the lagrangian $\widehat{\mathcal{L}}_{A}+\mathcal{L}_{\phi}+\widehat{\mathcal{L}}_{\mathrm{WZ}}+\widehat{\mathcal{L}}_{\psi}$.

Without any essential modification, our discussion in this section is applicable to the anomalous gauge theory with the gauge group $G \times \mathrm{U}(1)$ whenever the generators, $T_{a}$, of $G$ satisfy the conditions $\operatorname{tr}\left[T_{a}\right]=0$ and $\operatorname{tr}\left[T_{a}\left\{T_{b}, T_{c}\right\}\right]=0[16]$. For example, the generators of $\mathrm{SO}(n)(n \geq 3, n \neq 6)$ satisfy these conditions.

\section{Summary and Discussion}

In this paper we have studied anomalous gauge theories in four and six dimensions that contain antisymmetric tensor fields. It has been shown that in the 
anomalous $\mathrm{U}(1)$ and $\mathrm{SU}(2) \times \mathrm{U}(1)$ gauge theories in four dimensions, the $B F$ term with an antisymmetric tensor field $B_{\mu \nu}$ plays the roles of the WZ terms as well as the mass term of the $\mathrm{U}(1)$ gauge field $A_{\mu}$ by imposing suitable gauge transformation rules on $B_{\mu \nu}$. By virtue of the $B F$ term, the chiral anomalies are canceled and the gauge symmetries are recovered to the theories.

We have demonstrated, both at the classical and quantum levels, that the four-dimensional anomalous $\mathrm{U}(1)$ gauge theory with $B F$ term is dual to the four-dimensional anomalous U(1) gauge theory with a WZ term and with a Stückelberg type mass term of $A_{\mu}$. Similar duality has also been discussed in the six-dimensional anomalous $\mathrm{U}(1)$ gauge theory with $B F$ term and in the four-dimensional anomalous $\mathrm{SU}(2) \times \mathrm{U}(1)$ gauge theory with $B F$ term.

In six dimensions, we have considered the anomalous $\mathrm{U}(1)$ gauge theory with $B F^{2}$ term. This theory is substantially different from the six-dimensional anomalous $\mathrm{U}(1)$ gauge theory with $B F$ term, since the $B F$ term functions both as the WZ term and as the mass term of $A_{\mu}$, while the $B F^{2}$ term functions only as the WZ term. In each theory, the $B F$ or $B F^{2}$ term restores the U(1) gauge symmetry at the quantum level.

The $B F$ terms are known as generalizations of the Chern-Simons term in three dimensions; the anomalous gauge theories with $B F$ terms might be formulated to be higher-dimensional analogs of the three-dimensional anomalous gauge theory in which the Chern-Simons term restores the gauge symmetry at the quantum level [17].

Power-counting renormalizability of the four-dimensional anomalous $\mathrm{U}(1)$ gauge theory with WZ term is spoiled because of the WZ term, since it has a proportional constant with dimensions of length. On the other hand, the $B F$ term has a proportional constant with dimensions of mass, although it functions as the WZ term. This is desirable to renormalizability of the fourdimensional anomalous U(1) gauge theory with $B F$ term. However, instead of the $B F$ term, the Chern-Simons three-form included in the field strength $H_{\mu \nu \rho}$ has a proportional constant with dimensions of length. Consequently, the fourdimensional anomalous $\mathrm{U}(1)$ gauge theory with $B F$ term is also power-counting nonrenormalizable. The same can be said of the four-dimensional anomalous $\mathrm{SU}(2) \times \mathrm{U}(1)$ gauge theory with $B F$ term.

The anomalous $\mathrm{SU}(2) \times \mathrm{U}(1)$ gauge theory with $B F$ term seems to be applicable to constructing an electroweak model in which anomalies due to quarks and leptons do not cancel among these particles. An anomalous $\mathrm{SU}(2) \times \mathrm{U}(1)$ gauge theory with WZ term has indeed be applied to the description of electroweak model that lacks the top quark [18]. In our theory, however, only the $\mathrm{U}(1)$ gauge field $A_{\mu}$ becomes massive, while the $\mathrm{SU}(2)$ gauge field $\widehat{A}_{\mu}$ remains massless. This is due to the fact that the only abelian antisymmetric tensor field $B_{\mu \nu}$ has been introduced into the theory. The gauge fields $A_{\mu}$ and $\widehat{A}_{\mu}$ (or their linear combinations) can not be identified with the gauge bosons in the Weinberg-Salam model without considering further physical degrees of freedom. 
If we describe those gauge bosons in terms of the anomalous $\mathrm{SU}(2) \times \mathrm{U}(1)$ gauge theory with $B F$ term, it will be necessary to introduce non-abelian antisymmetric tensor fields of rank two [8] and to consider their interactions with the gauge fields $A_{\mu}$ and $\widehat{A}_{\mu}$.

\section{Acknowledgements}

We are grateful to Professor S. Naka and other members of the Theoretical Physics Group at Nihon University for their encouragements and useful comments. This work was supported in part by Nihon University Research Grant.

\section{References}

1. R. Jackiw and R. Rajaraman, Phys. Rev. Lett. 54, 1219 (1985); 54, 2060(E) (1985).

2. N. V. Krasnikov, Nuovo Cimento 89A, 308 (1985); 95A, 325 (1986).

I. G. Halliday, E. Rabinovici, A. Schwimmer and M. Chanowitz, Nucl. Phys. B268, 413 (1986).

M. S. Chanowitz, Phys. Lett. B171, 280 (1986).

S. Miyake and K. Shizuya, Phys. Rev. D36, 3781 (1987); Phys. Rev. D37, 2282 (1988).

3. O. Babelon, F. A. Schaposnik and C. M. Viallet, Phys. Lett. B177, 385 (1986).

K. Harada and I. Tsutsui, Phys. Lett. B183, 311 (1987); Prog. Theor. Phys. 78, 878 (1987).

4. L. D. Faddeev and S. L. Shatashvili, Phys. Lett. B167, 225 (1986).

5. R. Rajaraman, Phys. Lett. B184, 369 (1987).

A. Andrianov, A. Bassetto and R. Soldati, Phys. Rev. Lett. 63, 1554 (1989).

S. Miyake and K. Shizuya, Mod. Phys. Lett. A4, 2675 (1989).

6. A. Della Selva, L. Masperi and G. Thompson, Phys. Rev. D37, 2347 (1988).

T. Fujiwara, Y. Igarashi and J. Kubo, Nucl. Phys. B341, 695 (1990). 
7. E. Cremmer and J. Scherk, Nucl. Phys. B72, 117 (1974).

A. Aurilia and Y. Takahashi, Prog. Theor. Phys. 66, 693 (1981).

I. Oda and S. Yahikozawa, Prog. Theor. Phys. 83, 991 (1990).

T. J. Allen, M. J. Bowick and A. Lahiri, Mod. Phys. Lett. A6, 559 (1991).

A. Lahiri, Mod. Phys. Lett. A8, 2403 (1993).

H. Sawayanagi, Mod. Phys. Lett. A10, 813 (1995).

R. Amorim and J. Barcelos-Neto, Mod. Phys. Lett. A10, 917 (1995).

J. Barcelos-Neto and A. Cabo, Z. Phys. C74, 731 (1997).

8. D. Z. Freedman and P. K. Townsend, Nucl. Phys. B177, 282 (1981).

J. Barcelos-Neto, A. Cabo and M. B. D. Silva, Z. Phys. C72, 345 (1996).

D. S. Hwang and C. Y. Lee, J. Math. Phys. 38, 30 (1997).

A. Lahiri, Phys. Rev. D55, 5045 (1997).

J. Barcelos-Neto and S. Rabello, Z. Phys. C74, 715 (1997).

9. E. Bergshoeff, M. de Roo, B. de Wit and P. van Nieuwenhuizen, Nucl. Phys. B195, 97 (1982).

10. M. B. Green, J. H. Schwarz and E. Witten, Superstring Theory Vol. 2 (Cambridge University Press, New York, 1987)

11. T. Kimura, Prog. Theor. Phys. 64, 357 (1980).

H. Hata, T. Kugo and N. Ohta, Nucl. Phys. B178, 527 (1981).

J. Thierry-Mieg and L. Baulieu, Nucl. Phys. B228, 259 (1983).

M. Henneaux and C. Teitelboim, Quantization of Gauge Systems (Princeton University Press, New Jersey, 1992).

12. R. A. Bertlmann, Anomalies in Quantum Field Theory (Oxford University Press, New York, 1996).

13. G. F. Chapline and N. S. Manton, Phys. Lett. B120, 105 (1983).

14. S. Deguchi and T. Nakajima, Mod. Phys. Lett. A12, 111 (1997).

15. L. Baulieu, Phys. Lett. B126, 455 (1983); Nucl. Phys. B227, 157 (1983).

16. H. Georgi and S. L. Glashow, Phys. Rev. D6, 429 (1972). 
17. A. N. Redlich, Phys. Rev. Lett. 52, 18 (1984); Phys. Rev. D29, 2366 (1984).

18. T. Fujiwara and S. Kitakado, Mod. Phys. Lett. A8, 1639 (1993). 